\title{
UNA RECONSTRUCCIÓN FUNCIONAL DE LA CONDUCTA DE JUGAR: UN ESTUDIO DE CASO APLICADO AL TRASTORNO DE ASPERGER
}

\author{
Franclsco José Alós Cívlco* \\ José Jullo Carnerero Roldán** \\ -Universidad de Códoba \\ " Centro de Alta Eficacia para la Enseñanza del Lenguaje. Al Múdaris
}

\section{RESUMEN}

El propósito de este trabajo consiste en la adquisición, desarrollo y fortalecimiento de las interacciones verbales en la conducta de jugar con otras personas, de un niño diagnosticado con Trastomo de Asperger. La conducta de jugar podría ser conceptualizada como una conducta compleja, donde puede ocurrir cualquiera de los repertorios de conducta del niño. El trabajo enfatiza la necesidad de hacer un análisis detallado desde Conducta Verbal, de los elementos que participan en la interacción de juego. A la luz de los resultados, se observa que la aplicación de consecuencias deliberadas sobre comportamientos definidos operacionalmente, tienen efectos diferenciales para la adquisición y generalización de las conductas que forman las secuencias de juego.

Correspondencia: Francisco José Alós Civico. Facultad de Cienclas de la Educación. Departamento de Educeción. Area de Personalidad, Evaluación y Tratamiento Psicológicos. Universidad de Córdoba. Avda. San Alberto Magno s/n. 14071 Córdoba (España). Teléfono: (957)211021. E-mail: od1alcif uco.es.

Los autores quieren dar las gracias a Juan Antonio Moriana por sus excelentes comentarios y sugerencies hechas al manuscrito original y a Jorge Ojeda por su ayuda en el diseño y realización de Jas gráficas. 
En el periodo de seguimiento se evidencia el mantenimiento de los niveles de adquisición tres meses después de alcanzar el criterio meta, para cada una de las cuatro conductas seleccionadas.

Palabras clavo: SfNDROME DE ASPERGER, CONDUCTA VERBAL, CONDUCTA DE JUGAR, ANÁLISIS FUNCIONAL, GENERALIZACIÓN.

\section{SUMMARY}

The purpose of this work consists on the acquisition, development and invigoration of the verbal interactions in the behavior of playing with other people, of a boy diagnosed with Dysfunction of Asperger. The behavior of playing could be conceptualized as a complex behavior, where they can happen anyone of the boy's repertoires of behavior. Our work emphasizes the necessity to make a detailed analysis from Verbal Behavior of the elements that participate in the game interaction. By the light of the results, it is observed that the applicatlon of deliberate consequences on defined behaviors operationally, they have differential effects for the acqulsition and generalization of the behaviors that form the game sequences.

In the period of follow-up the maintenance of the levels of acquisition is evidenced three months after reaching the approach it puts, for each one of the four selected behaviors.

Key wOrds: SYNDROME OF ASPERGER, VERBAL BEHAVOR, BEHAVOR OF PLAYNG, FUNCTIONAL ANALYSIS, GENERALLZATION.

\section{INTRODUCCIÓN}

EI DSM-IV-TR (APA, 2002) afima que "las características esenciales del trastorno de Asperger son la incapacidad grave y permanente para la interacción social, y la presencia de pautas de conducta, intereses y actividades repetitivos y restringidos. El trastorno puede causar insuficiencias clínicamente significativas en la vida social y laboral, as! 
como en otras áreas Importantes de la actlvidad del individuo. En contraste con el trastorno autista, no hay retrasos clinicamente significativos $\mathrm{nl}$ alteraciones en la adquisición del lenguaje". (pag 91).

Según Willams (1995) existen siete características destacadas que pueden describir el comportamiento de estas personas en el contexto escolar:

1. Se puede advertir persistencia en determinadas rutinas.

2. Manifiestan limitaciones para comprender de forma efectiva las relaciones humanas, con una especial dificultad para el aprendizaje de las reglas sociales convencionales.

3. Pueden presentar un abanico restringido de intereses, con la presencia de preocupaciones excéntricas o intereses extraños.

4. Se puede observar en algunos casos, falta de focalización para determinadas actividades escolares.

5. Limitaciones en la coordinación motora.

6. Dificultades en algunas áreas académicas.

7. Pueden no poseer los recursos emocionales pertinentes para adaptarse y responder a algunas demandas sociales.

No obstante cada niño/a posee sus propias peculiaridades idiosincrásicas y estas características "tipicas" se manifiestan de forma diferente en cada una de estas personas (Williams, 1995; Klin y Volkmar 1995). Pero sea como fuere, algunos profesionales tienen tendencia a la categonización, en tanto que la descripción de características comunes a un grupo de personas. Una polémica vigente en la actualidad, es la determinación de categorías clasificatorias que pudieran dar cuenta de los comportamientos presentados por diferentes colectivos. A partir del trabajo de Wing y Gould (1979), se ha desarrollado el concepto de espectro autista, utilizado como un nuevo descriptor en el cual se abandona la idea de la exclusión categorial entre diferentes trastornos, para enfatizar que los rasgos autistas pueden ser situados en un conjunto de dimensiones que no son exclusivas solo en el autismo, sino que pueden estar presentes, en diferentes medidas, en otros trastomos (Rivière, 1997). Actualmente, todavía no existe unanimidad en relación a qué trastomos deben ser incluidos bajo el descriptor de espectro y cual debe ser la amplitud del mismo (Martos, 2001). De igual forma, aún no está clara la díferencia que existe entre el trastomo de Asperger y el autismo de alto nivel de 
funcionamiento (Martos, 2001), aunque algunos estudios han enfatizado una distinción entre ambos, la mayorla de las investigaciones no han podido encontrar ninguna diferencia significativa entre dichos grupos. (Bauer, 1996; Baron-Cohen, Wheelwright, Spong, Scahill y Lawson, 2001). En cualquier caso, la incidencia del Síndrome de Asperger (S.A), se ha valorado en torno a 20-25 casos por cada 10.000 personas, frente a 4 casos por cada 10.000 para el autismo clásico de Kanner (Bauer, 1996).

Algunas de las razones esgnimidas para el mantenimiento de esta categorla (S.A.) estriba en que parece existir un mejor pronóstico respecto al autismo típico. De igual manera, según Bishop (1989) esta etiqueta goza de una mayor aceptación entre los padres y profesionales. Nos encontramos desde nuestro punto de vista, ante uno de los problemas de desarrollo con mejor pronóstico terapéutico, descrito por una alta probabilidad en el progreso irreversible del desarrollo psicológico dentro de un continuo en la dirección de la normalidad, que cuestiona y advierte a los profesionales de la extremada prudencia que se debe tener a la hora de estimar los posibles límites en el desarrollo psicológico que una persona con estas caracteristicas puede tener.

En la actualidad existen experiencias de personas que fueron en algún momento diagnosticados con este problema, que han llegado a alcanzar niveles de conducta verbal altamente complejos e inclusión social, sirva de botón de muestra las palabras de Roger Niwson (2001): "Me diagnosticaron autismo de tipo moderado en las primeras semanas de vida. Ahora, este diagnóstico es menos obvio de lo que fue entonces. Personas de otros países y otras culturas suelen comentar que ahora ya no parezco autista. Sin embargo, la gente de mi propio país y de mi propia cultura, con frecuencia notan que soy un poco diferente al resto de personas"(pag. 267). Estas experiencias nos alientan para trabajar y desarrollar procedimientos que permitan adquirir habilidades, conocimientos y destrezas funcionales, dando desde nuestra perspectiva prioridad a la solución de problemas frente a la especulación teórica. De igual manera, existen investigaciones que están poniendo de manifiesto unos extraordinarios resultados con estos colectivos (Williams, 1998; Williams, Donley y Keller, 2000; Pérez-González y Williams, 2002), línea de investigación que destaca por la coherencia entre los supuestos conceptuales y el desarrollo de procedimientos 
prácticos, por la vertebración del aprendizaje a través de conducta verbal (Skinner, 1981), más el enniquecimiento de la tecnología de aprendizaje de las relaciones de estímulos, diseño de procedimientos sistemáticos para la enseñanza, gradación jerárquica del currículo a enseñar, funcionalidad del mismo, implicación y coordinación de todos los agentes relevantes para el aprendizaje de este grupo humano.

Se describe seguidamente la intervención efectuada para desarrollar la conducta de jugar de un chico diagnosticado con trastorno de Asperger. La conducta de jugar generalmente se engloba en el área social, aunque la asignación de un comportamiento a un área del desarrollo u otro, vendrá determinado por el análisis que se haga de los elementos, variables y conductas necesarias para llegar a adquinir dicha habilidad, la conducta de jugar bien puede considerarse dentro del área cognitiva, emocional, social o conjuntamente en más de un área. Ahora bien, su conceptualización varla según el posicionamiento teónico que enmarque el análisis de este evento, aunque la mayorla de las aproximaciones explicativas parecen coincidir en la necesidad de desarrollar y fortalecer este repertorio, debido entre otras razones a su pertinencia para el desarrollo posterior de habilidades sociales.

El planteamiento de la presente intervención se centra en el análisis de las funciones de los distintos eslabones conductuales implicados en las interacciones de juego, para determinar cuales son las variables que permiten que se desarrollen secuencias efectivas de juego. De aqul la necesidad de hacer un análisis de las consecuencias (automáticas y/o sociales) (Luciano, 1989) que están implicadas en dichas interacciones. Desde esta aproximación descriptiva, en el juego pueden ocurrir cualquiera de los repertorios conductuales del niño (motor y/o verbal), de forma abierta y/o cubierta, y todo ello bajo secuencias de comportamientos simultáneos o sucesivos (Bijou, 1982).

\section{MÉTOdO}

\section{Sujeto}

NAL es un chico que en el momento de la intervención contaba con 7 años de edad y habla sido diagnosticado con el síndrome de Asperger 
(por profesionales ajenos a la investigación). En la actualidad se encuentra escolarizado en un centro de integración escolar.

De las diferentes evaluaciones realizadas hasta la fecha se deduce que el desarrollo psicomotriz, el lenguaje y capacidad cognitiva son acordes a la edad cronológica del niño. Por tanto, desde el punto de vista normativo se podría afirmar que sus puntuaciones se encuentran ubicadas dentro de la curva que recoge los puntajes considerados como "normales".

Los padres demandan ante todo la necesidad de trabajar en fortalecer las interacciones de juego con iguales. Plantean que cuando su hijo se encuentra en el colegio o en casa con otros niños y se inicia un juego en el cual como mínimo hay dos participantes, NAL acaba jugando "solo y se aisla". Todo esto hace que disponga de pocas oportunidades para relacionarse con otros niños/as, lo cual les preocupa de cara al futuro, pues con el paso del tiempo, saben que dichas interacciones suelen ser bastantes frecuentes.

Se constata que NAL desarrolla juego simbólico e imaginativo, pero de la observación no sistemática, se deduce que el juego es repetitivo y siempre que hay una situación libre en la que se le da la posibilidad para decidir entre varios juegos elige:

a) Jugar a cocinar: se utilizan diferentes figuras de plástico que representan frutas y alimentos, una caja de cartón que sirve para batir cual si fuera una batidora o calentar como un grill. Se desarrolla una secuencia de juego donde se preparan diferentes alimentos para posteriormente ser comidos.

b) Jugar a las casas: Se utiliza un órgano musical a modo de portero automático para acceder a la casa y también se emplea como numeración de un ascensor. Adoptando NALel papel de adulto (padre) y el adulto es instruido para interpretar el papel de niño (hijo).

En los dos juegos, el adulto sólo participa en contadas ocasiones, siendo el desarrollo argumental del juego planteado por NAL. Se advierte que el niño muestra resistencia al cambio del argumento, cuando éste es planteado por el adulto, siguiendo corno no podía ser de otra manera con la trama original. De la observación de estas mismas actividades con un hermano menor de 5 años, se detecta que a poco del inicio de las interacciones estudiadas, cada uno de los dos niños juega individualmente, sollcitando el pequeño la participación del observador 
no participante, para que su hermano le deje colaborar o simplemente inicia otra acción.

De la interacción con el padre en un juego (parchís), se detecta que es ahora el adulto el que dirige y organiza las secuencias, haciendo que el niño se ajuste a las normas establecidas, a través de las continuas instrucciones y correcciones sobre el comportamiento efectuado por NAL. Todo lo cual pone de marifiesto que en una situación en la cual existe mayor estructuración (siempre más probable con adultos) se presentarán en menor cuantía los aspectos problemáticos de la interacción en el juego, cosa por otro lado menos probable con iguales. Consideraciones que son corroboradas y coinciden con las descripciones hechas por los padres, donde se afirma que con los adultos, NAL tiene menor dificultad para jugar.

Por último y relacionado con otros aspectos del repertorio conductual del niño, los padres informan que NAL no suele preguntar cuando mantienen una conversación, de igual manera se observa una especial predilección por aquellos juegos individuales que giran sobre sí mismos a gran velocidad (peonza y tiovivo), haciéndolos girar durante varios minutos consecutivos sin parar.

\section{Procedimiento}

Se aplicó un diseño de caso único de cambio de criterio (Barlow y Hersen, 1988) a través de conductas, con seguimiento a los tres meses.

\section{Evaluación}

En la evaluación que se describe seguidamente, el objetivo era medir el nivel adquinido para diferentes interacciones conductuales por NAL en situaciones naturales y comunes, en las cuales aparecen secuencias de juego con otras personas. Se debe afirmar que esta evaluación centrada en conducta verbal, permitió posteriormente analizar los repertorios verbales implicados en las unidades de conversación (Greer, 2002) necesarias para mantener una interacción socialmente adaptativa de juego (con otras personas).

La evaluación se realizó para interacciones de juego entre:

a) Niño-Niño (Un niño/a y NAL). 
b) Adulto-Niño (Un adulto y NAL).

La evaluación se llevó a cabo en una habitación habilitada para tal fin, se seleccionaron tres juegos: 1) parchis; 2) construcciones de figuras con piezas, cartulina y plastilina y 3 ) uso de objetos con ficción -juegos simbólicos- (se utilizan diferentes materiales para representar otros objetos o animales, por ejemplo una silla representa un caballo en el juego de vaqueros) en los que los intervalos de evaluación duraron quince minutos por juego. Las respuestas se iban anotando en una hoja de registro y no se administraba consecuencias deliberadas sobre las conductas implicadas. De esta forma se seleccionaron cuatro interacciones conductuales a registrar:

1. Respuestas verbales de NAL ante órdenes:

a) NAL juega con una niña al parchís. Porcentaje de ensayos: $40 \%$ Correctos y $60 \%$ Incorrectos.

b) NAL juega con un adulto al parchís. Porcentaje de ensayos: $0 \%$ Correctos y $100 \%$ incorrectos.

- NAL juega con un adulto a construir una figura (robot) con piezas. Porcentaje de ensayos: $52,6 \%$ Correctos y $47,4 \%$ incorrectos.

- NAL juega con un adulto a un juego simbólico (vaqueros). Porcentaje de ensayos: $27,3 \%$ Correctos y $72,7 \%$ incorrectos.

2. Seguimiento de instrucciones de NAL:

a) NAL juega con un niño a construir un robot con piezas. Porcentaje de ensayos: $25 \%$ Correctos y $\mathbf{7 5} \%$ Incorrectos.

b) NAL juega con un adulto al parchís. Porcentaje de ensayos: 16,7 $\%$ Correctos y $83,3 \%$ incorrectos.

- NAL juega con un adulto a construir una figura (robot) con piezas. Porcentaje de ensayos: $14,3 \%$ Correctos y $85,7 \%$ incorrectos.

- NAL juega con un adulto a un juego simbólico (vaqueros). Porcentaje de ensayos: $25 \%$ Correctos y $75 \%$ Incorrectos.

3. Preguntas iniciadas por NAL:

a) NAL juega con una niña a un juego simbólico (espadachines). Tasa (frecuencia de ocurrencia de la conducta dividida por el tiempo transcurrido en el juego): 0'06 al minuto.

b) NAL juega con un adulto al parchís. Tasa: 0'13 al minuto.

- NAL juega con un adulto a construir una figura (robot) con piezas. Tasa: 0'13 al minuto. 
- NAL juega con un adulto a un juego simbólico (vaqueros). Tasa: 0 '13 al minuto.

4. Transgresión de NAL al orden según los turnos:

a) NAL juega con un niño al parchís: Tasa: 1 '7 al minuto.

b) NAL juega con un adulto al parchís. Tasa: 1'74 al minuto.

- NAL juega con un adulto a construir una figura (robot) con piezas. Tasa: 1'14 al minuto.

- NAL juega con un adulto a un juego simbólico (vaqueros). Tasa: $1 ' 27$ al minuto.

\section{Análisis funcional}

El presente análisis funcional se hace sobre episodios verbales y no verbales. En base a la evaluación, se pretende aislar los eslabones que están interviniendo en el comportamiento actual de juego del niño para posteriormente estructurar la intervención.

Del total de conductas que pueden ocurrir en el juego se eligieron las siguientes interacciones conductuales:

1. Respuesta verbal del niño ante órdenes: ante la estimulación verbal de una persona que implica la realización de una actividad, el sujeto debe emite una respuesta verbal audible, que confirma o deniega la instrucción verbal.

Ejemplo: un compañero de juego le dice: dame la pelota (estímulo discriminativo) y el niño puede responder afirmativa o negativamente: sí, vale, bueno, no, después, etc (respuesta verbal y consecuencia social).

2. Seguimiento de instrucciones: emisión antecedente de estimulación verbal que implica la realización de conducta no verbal por el niño que actúa como audiencia, existiendo una correspondencia entre lo que se le ha dicho que haga y lo que ha hecho, lo cual implica una discriminación condicional de segundo orden. Por ejemplo en una mesa se encuentra un lápiz y una goma, el maestro le dice al niño: (recoge el lápiz) (estímulo contextual) recoge, (estímulo condicional) lápiz, los objetos (el lápiz actúa como estímulo discriminativo y la goma como estímulo delta), la respuesta es recoger el objeto y las consecuencias sociales (bien, genial, etc) serán dispensadas por el adulto. 
3. El niño inicia Preguntas: emisión antecedente de estimulación verbal de forma interrogativa que solicita respuestas verbales vocales a un oyente y dichas respuestas satisfacen las demandas hechas por NAL.

El niño observa la mano cerrada del adulto (estímulo discriminativo) y dice: ¿Qué tienes en la mano? (respuesta y estimulo discriminativo para la siguiente respuesta del adulto). Tengo un trozo de plastilina (respuesta verbal y consecuencias sociales).

4. Respeto al turno: interacción de las conductas motoras de dos personas, de forma serial, alterna y encadenada.

Por ejemplo, en el parchís, una persona tira el dado (estimulo discriminativo para la siguiente respuesta de mover la ficha), mover la ficha por parte de la primera persona, es el estímulo discriminativo para que el otro jugador tire el dado.

\section{Intervenclón}

El chico participa en un programa extraescolar de enseñanza individualizada (alumno-maestro) de una hora de duración dos veces por semana, donde las unidades de enseñanza abarcaban diferentes programas (académicos y sociales), entre los cuales se incluye la intervención específica sobre el tema que nos ocupa.

El programa de la intervención para el juego, se produjo bajo una secuencia de interacción sin interrupción entre el niño y el adulto, con una duración variable entre 15 y 30 minutos, en una sala habilitada para tal fin. Los juegos habituales utilizados para el entrenamiento fueron: 1) Juego del parchis y 2) Construcciones de figuras y objetos con diferentes materiales (plastilina, papel, palillos y piezas de lego). Las respuestas eran anotadas por el maestro en una hoja de registro y contingentemente se administraban las consecuencias (sociales $y$ fichas).

Se seleccionaron cuatro objetivos a trabajar de forma simultánea en las interacciones ocurridas en el juego: 1) Fortalecimiento de las respuestas verbales de NAL ante órdenes, 2) Fortalecimiento de las conductas de seguimiento de instrucciones, 3) Fortalecimiento de preguntas iniciadas por NAL y 4) Eliminación de las conductas de transgresión al orden según los turnos. 
1. Fortalecimiento de las respuestas verbales de NAL ante órdenes: Por ejemplo, en el parchís, el maestro dice: "tira" (estímulo discriminativo) y el niño responde: "vale" (respuesta verbal) y se administran consecuencias sociales por parte del maestro (muy bien, genial, etc) acompañadas de una ficha para ser canjeada al término de la sesión por un regalo sorpresa (chucherías) seleccionado dependiendo de las preferencias del niño. Cuando una respuesta va seguida de una consecuencia y debido a esta presentación hace que la operante incremente o se mantenga, entonces técnicamente se ha producido un procedimiento de reforzamiento positivo.

Una vez alcanzado el criterio meta en la sesión 13, se introdujo una fase de mantenimiento desde la sesión 14 a la 21, ahora las conductas fueron sometidas a un programa de RV3 desde las sesiones 14 hasta 18 y RV4 para las dos siguientes. Desde las sesiones 22 a la 25 se produjo el entrenamiento para la generalización, en la sesión 22, NAL y un niño juegan al juego entrenado (parchís), en la 23 NAL y el adulto juegan a un nuevo juego (futbolín), en la 24 NAL y el adulto juegan en un juego no entrenado en un contexto nuevo (fútbol), y en la 25 NAL y un nuevo adulto interaccionan en un juego no entrenado (minigolf).

2. Fortalecimiento de las conductas de seguimiento de instrucciones: Por ejemplo, el adulto le dice al niño: "coge la pelota", (estímulo contextual) "coge", (estímulo condicional) "pelota", la pelota (objeto) (es el estímulo discriminativo) y el niño realiza la conducta de recoger la pelota (respuesta) por lo que recibe consecuencias sociales (muy bien, genial, etc) y la obtención de una ficha. En este caso el porcentaje de ensayos correctos es mantenido o incrementado por la aplicación contingente de estas consecuencias, por lo que se produce un proceso de reforzamiento positivo.

Una vez alcanzado el criterio meta en la sesión 9, se introdujo una fase de mantenimiento desde las sesiones 11 a la 21 , donde las conductas fueron sometidas a un programa de RF1. Desde las sesiones 23 a la 25 se produjo el entrenamiento para la generalización, en la 23 NAL y el adulto juegan al nuevo juego (futbolín), en la 24 NAL y el adulto juegan en un juego no entrenado en un contexto nuevo (fútbol), y en la 25 NAL y un nuevo adulto interaccionan en un juego no entrenado (minigolf).

3. Fortalecimiento de preguntas iniciadas por NAL: Por ejemplo, en el parchís, el adulto tira el dado (estímulo discriminativo) y el niño dice: 
"¿Qué te ha tocado?" (respuesta y estímulo discriminativo para la nueva respuesta del adulto) y el maestro responde: "Me ha tocado un seis" (respuesta verbal y consecuencias sociales) y se entrega una ficha. La aplicación contingente de una consecuencia lleva al incremento o mantenimiento de la tasa de respuesta.

Una vez alcanzado el criterio meta en la sesión 8 , se introdujo una fase de mantenimiento desde la sesión 9 a la 20, donde las conductas fueron sometidas a un programa de RF1. Desde las sesiones 23 a la 25 se produjo el entrenamiento para la generalización, en la 23 NAL y el adulto juegan a un nuevo juego (futbolín), en la $25 \mathrm{NAL}$ y el adulto juegan en un juego no entrenado en un contexto nuevo (fútbol), y en la 25 NAL y un nuevo adulto interaccionan en un juego no entrenado (minigolf).

4. Eliminación de las conductas de transgresión al orden según los turnos: Considerábamos como una transgresión en el parchis cuando el maestro tira el dado (estímulo discriminativo), el niño coge la ficha del adulto y mueve con ella (respuesta). Haciendo un análisis pormenorizado se podría afirmar que la respuesta del adulto (mover la ficha), es la que se debía convertir en un estímulo discriminativo para la respuesta de tirar el dado por el niño. La intervención desarrollada consistía en que cada vez que ocurría una secuencia errónea (transgresora), se le retiraba al niño dos de los puntos que ya tiene en su haber, por lo que técnicamente se estaría produciendo una retirada del reforzador que el sujeto previamente había obtenido (castigo en forma de coste de respuesta)

Una vez alcanzado el criterio meta en la sesión 17, se introdujo una fase de mantenimiento desde la sesión 19 a la 21, donde las conductas fueron sometidas a un programa de RF1. Desde las sesiones 22 a la 25 se produjo el entrenamiento para la generalización, en la sesión 22, NAL y un niño juegan al juego entrenado (parchís), en la 23 NAL y el adulto juegan al nuevo juego (futbolín), en la 24 NAL y el adulto juegan en un juego no entrenado en un contexto nuevo (fútbol), y en la $25 \mathrm{NAL}$ $y$ un nuevo adulto interaccionan en un juego no entrenado (minigolf).

El seguimiento para las cuatro conductas, se realizó tres meses después (sesión 26) de la finalización de la fase anterior, de nuevo en el contexto extraescolar transcurridos los tres meses de verano, el maestro habitual del programa de entrenamiento evaluó los niveles de las cuatro interacciones conductuales en el juego del parchís. 


\section{RESULTADOS}

1. Para las respuestas verbales del niño ante órdenes se alcanza el objetivo en la sesión 13. Se consideró como criterio meta, tres sesiones consecutivas (igual o superior a un $80 \%$ ) de ensayos correctos.

- Se advierten porcentajes superiores al $80 \%$ en el entrenamiento en generalización para las siguientes condiciones: a. NAL y un niño jugando a un juego entrenado (parchís), b. NAL y el adulto jugando a un nuevo juego (futbolín), c. NAL y el adulto jugando en un juego no entrenado en un contexto nuevo (fútbol), d. NAL y un nuevo adulto en un juego no entrenado (minigolf). De igual manera se evidencia el mantenimiento en el periodo de seguimiento de los criterios de ejecución tomados para la conducta objetivo, tres meses después de haber alcanzado el criterio meta.

2. Para el seguimiento de instrucciones se alcanza el objetivo en la sesión 9. Se consideró como criterio meta, tres sesiones consecutivas igual o superior a un $80 \%$ de ensayos correctos.

- Se observan porcentajes superiores al $80 \%$ en el entrenamiento en generalización para las siguientes condiciones: $b$. NAL y el adulto jugando a un nuevo juego (futbolín), c. NAL y el adulto jugando en un juego no entrenado en un contexto nuevo (fútbol), $d$. NAL y un nuevo adulto en un juego no entrenado (minigolf). De igual manera se advierte el mantenimiento en el periodo de seguimiento de los criterios de ejecución tomados para la conducta objetivo, tres meses después de haber alcanzado el criterio meta.

3. Para las preguntas iniciadas por el niño se observa estabilidad en la curva de aprendizaje en la sesión 8 . Se determinó como criterio meta cinco sesiones consecutivas con una tasa de respuesta igual o superior a 0.3 .

- Se advierte una tasa alrededor de 0.3 en el entrenamiento en generalización para las siguientes condiciones: b. NAL y el adulto jugando a un nuevo juego (futbolín), c. NAL y el adulto jugando en un juego no entrenado en un contexto nuevo (fútbol). En el periodo de seguimiento se observa una tasa de 0.3, tres meses después de haber alcanzado el criterio meta.

4. Para la eliminación de la transgresión al orden según los tumos, el objetivo fue alcanzado en la sesión 17. Se consideró como criterio 
meta para este objetivo, cinco sesiones con una tasa (igual o inferior a 0.1) de conductas de transgresión al orden.

- Se advierte una tasa igual o inferior a 0.1 en el entrenamiento en generalización para las siguientes condiciones: a. NAL y el adulto jugando a un nuevo juego (futbolín), b. NAL y el adulto jugando en un juego no entrenado en un contexto nuevo (fútbol), c. NAL y un niño jugando al juego entrenado (parchis), d. NAL y un nuevo adulto en un juego no entrenado (minigolf). De igual manera se comprueba el mantenimiento en el periodo de seguimiento de los criterios de ejecución tomados para la conducta objetivo, tres meses después de haber alcanzado el criterio meta.
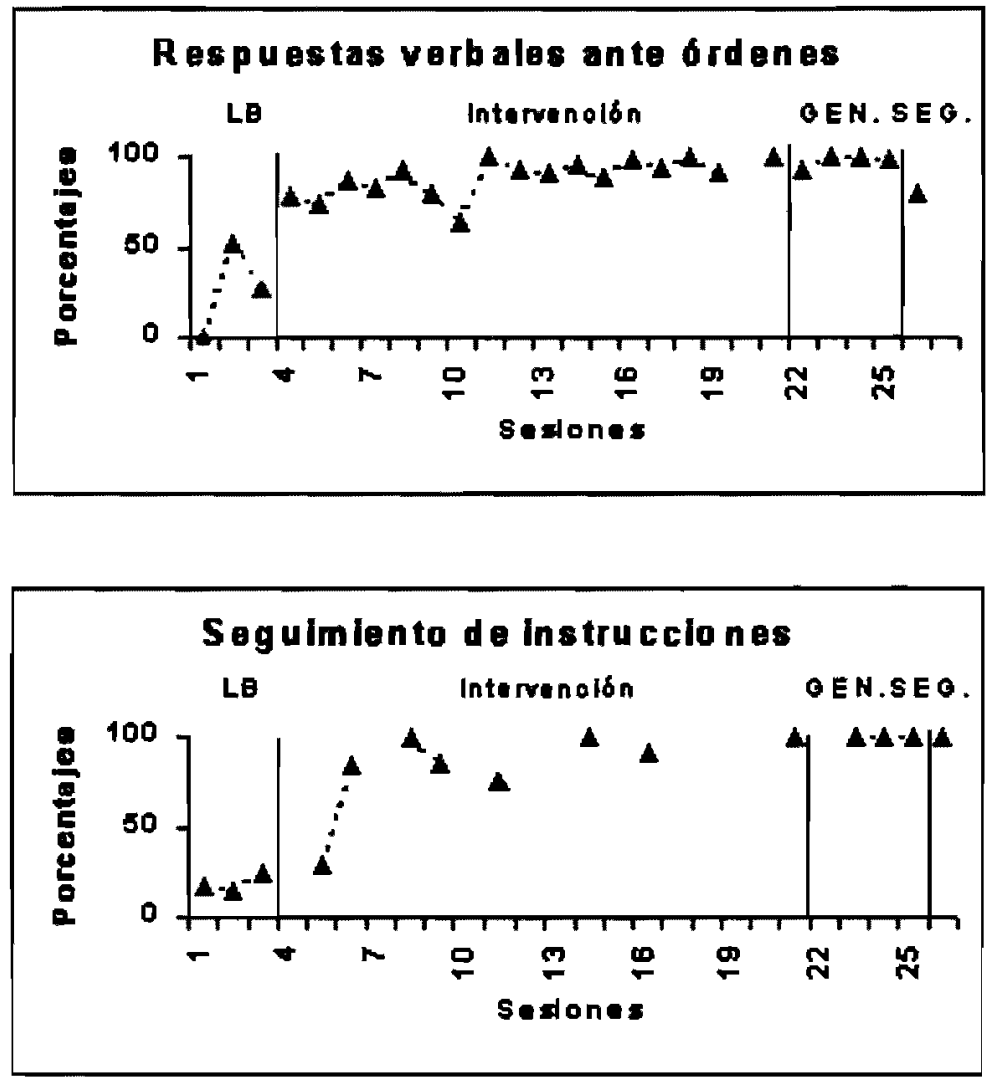

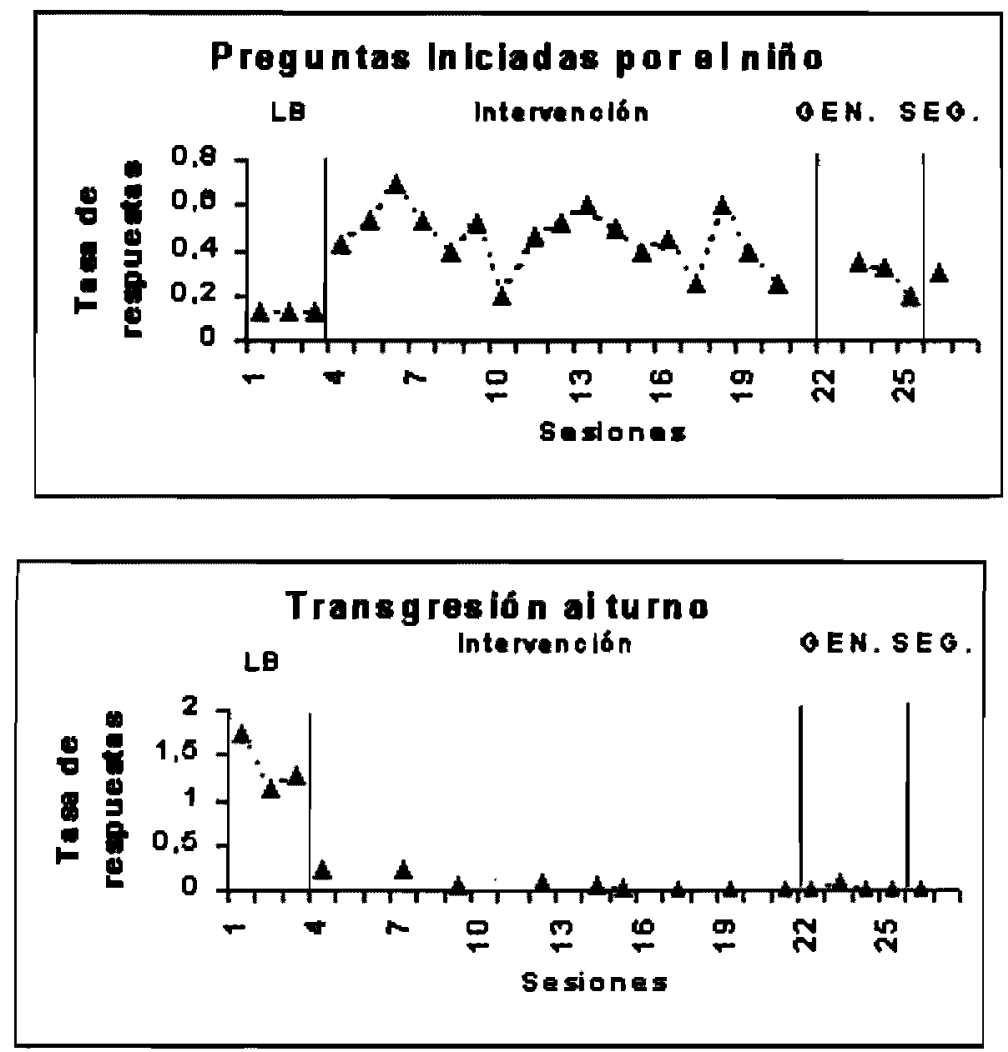

\section{DISCUSIÓN Y CONCLUSIONES}

Las personas con trastorno de Asperger presentan limitaciones en aspectos del área social (Bishop, 1989; Williams, 1995; Klin y Volkmar, 1995; Bauer, 1996; APA, 2000; Martos, 2001), por lo que el desarrollo de procedimientos que permitan el análisis e intervención en este campo supone una aportación necesaria y pertinente. En nuestra opinión, el repertorio necesario para desarrollar la habilidad de jugar está mediado socialmente. Un observador que describe una secuencia conductual (por ej. la manipulación de objetos) basándose en la topografía, podría llegar a hacer una clasificación de la conducta observada como una actividad de juego. Aunque lo realmente relevante, 
desde nuestro punto de vista, es determinar las funciones (sociales y/ o automáticas) que están implicadas en dicho juego. Esta afirmación sugiere que desde esta perspectiva se podría encontrar una jerarquía de tipos de juegos que iría desde aquellos con mayor grado de consecuencias automáticas hasta aquellos donde lo más relevante son las consecuencias sociales, cuya expresión máxima es el juego simbólico o de ficción. Pongamos por caso, que un niño puede estar interaccionando con un objeto y que las únicas consecuencias relevantes sean de tipo automático (en cualquiera de las modalidades sensoriales: auditivas, visuales, etc) y que esa manipulación del objeto va acompañada de una topografla o forma peculiar. Ante esto, un observador podría describir que el niño intencionadamente está jugando e incluso que juega a algo en concreto. El observador estaría "atribuyendo" unas funciones en base a su propia conducta verbal, no teniendo en cuenta el nivel de implicación de la conducta verbal del niño que "juega". Así pues, cualquier explicación que se haga sobre las dificultades o la imposibilidad de desarrollar juego simbólico en niños con algún Trastorno del Espectro Autista, debiera considerar un análisis del lenguaje, conceptualizado específicamente en este trabajo como conducta verbal (Skinner, 1981; Michael, 1982).

El presente artículo es una propuesta metodológica para la intervención efectiva en comportamientos complejos (conducta de jugar), la selección de interacciones conductuales (respuestas verbales, seguimiento de instrucciones, realización de preguntas y respeto de los turnos) permite explicitar secuencias discretas que forman parte de un conjunto de comportamientos encadenados que denominamos juego, lo cual implica el establecimiento de una categorización previa a la intervención y la aplicación de contingencias una vez establecidas las relaciones funcionales. A nuestro juicio, la conducta de jugar podría ser un excelente ejemplo de una secuencia compleja y encadenada de comportamientos, que en el presente estudio implica el comportarniento (verbal y no verbal) de dos personas (Bijou, 1982). La subdivisión de la conducta de jugar en unidades de análisis discretas y diferenciadas operacionalmente, permite intervenir sobre ellas para provocar de forma deliberada cambios en su ejecución.

Este trabajo pone de manifiesto que el problema en la intervención sobre este tipo de conductas complejas (conducta de jugar) 
principalmente estriba, en poder describir e intervenir a través del análisis funcional de las cadenas conductuales implicadas en estas interacciones, los datos indican que las respuestas del sujeto son susceptibles a la aplicación deliberada de contingencias.

La intervención fue vertebrada en todo momento por la interacción adulto-niño, para posteriormente ir propiciando la generalización a otras personas, juegos y contextos. La peculariedad del presente estudio de caso, estriba en que la categorización e intervención de los comportamientos seleccionados ocurren en una secuencia de juego real, por lo que el entrenamiento no se produce de manera aislado en programas independientes, esto supone que en la interacción de juego han de aparecer en alguna medida los comportamientos estudiados para poder ser fortalecidos o debilitados. De los datos disponibles se puede afirmar que los procedimientos utilizados son efectivos en los repertorios:

1. Respuestas verbales ante órdenes. Tras la intervención en 13 sesiones el chico alcanza el criterio meta, lo cual supone una rápida adquisición del repertorio entrenado.

2. Seguimiento de instrucciones. Tras la intervención en 4 sesiones se alcanza el criterio menta, este hecho supone que la respuesta de seguir instrucciones ha quedado rápidamente seleccionada por las consecuencias.

3. Respeto al turno. Tras la intervención en 7 sesiones se alcanza en criterio menta, este hecho supone que las respuestas de transgresión al turno han sido rápidamente afectadas por la aplicación del coste de respuesta.

4. En relación a la iniciación de preguntas por parte de NAL, se observa un discreto incremento en la interacción con el adulto y ringún cambio significativo con los niños (por lo que a la finalización del presente estudio, se propuso fortalecer cualquier pregunta iniciada por NAL en el contexto clínico o familiar), este repertorio a priori parece tener un mayor grado de complejidad que los restantes, por lo que los datos del presente estudio parecen indican que las respuestas son susceptibles a la aplicación de consecuencias, pero la dificultad de este repertorio (en tanto que un discreto incremento) está en que en esta habilidad, el chico tiene que aprender cuales son las condiciones adecuadas para la realización de preguntas, es decir, 
ante que conjunto de estímulos ha de realizar las preguntas, por lo que las condiciones antecedentes en este repertorio son más difusas que en las otras interacciones, dado lo cual, se hace pertinente desarrollar un programa especifico para fortalecer una clase de respuestas generalizadas de preguntar. Existen estudios en la literatura del análisis de la conducta, en los que se abordan de manera directa y planificada la enseñanza de habilidades de conducta verbal relacionadas con hacer preguntas, donde se les enseña a niños con autismo a hacer preguntar sobre objetos que están ocultos (Williams, Donley y Keller, 2000).

La generalización se establece para nuevos juegos, contextos y personas (adulto o niño). Ahora bien, es necesario señalar que para considerar técnicamente la aparición de la generalización de la respuesta ante un nuevo estímulo, la primera nueva relación de control funcional que ocurre no debe ser reforzada (Luciano, Herruzo y Barnes-Holmes, 2001), dado que por definición la generalización implica un nuevo control funcional producto de la historia del entrenamiento, pero en el caso que nos ocupa (un estudio de caso) y dada la frecuencia de los cornportamientos presentados, la no aplicación de consecuencias tras el nuevo control funcional, sería técnicamente la introducción de un proceso de extinción sobre la nueva interacción conductual, por lo que justamente se haría lo contrario del objetivo perseguido, por ello en el presente trabajo no se está comprobando experimentalmente el surgimiento de la generalización, sino que se estaría entrenando de forma aplicada la generalización.

En el periodo de seguimiento se observa el mantenimiento de las conductas a los tres meses de la adquisición, lo cual probabiliza la ocurrencia de dichos repertorios en los contextos naturales.

El presente estudio es una propuesta para la aplicación de un programa de enseñanza que incluye descripción, análisis, planificación $\theta$ intervención en una conducta compleja (jugar). El desarrollo de estudios de este tipo, podría ofrecer estrategias mensurables y medibles para incentivar la participación de personas con limitaciones en este ámbito (juego), en actividades fundamentalmente mediadas por interacciones sociales. 


\section{REFERENCIAS BIBLIOGRÁFICAS}

American Psychiatric Association (APA) (2002). Diagnóstico y manual estadístico de desordenes mentales. (DSM-IV-TR). Madrid: APA (Orig. 2000).

Barlow, D. H. y Hersen, M. (1988). Diseños experimentales de caso único. Estrategías para el estudio del cambio conductual. Barcelona: Martínez Roca (Orig. 1984).

Baron-Cogen, S., Wheelwright, S., Spong, A., Scahill, V., y Lawson, J. (2001). ¿Son independientes la física intuitiva y la psicología intuitiva? Un test con niños con síndrome de Asperger. En J. Martos y A. Rivière (Comps.) Autismo: comprensión y explicación actual (pp. 121-153) Madrid: Ministerio de Trabajo y Asuntos Sociales.

Bauer, S. (1996). Asperger Syndrome. Disponible en Internet: http // www.apennj.org/bauer.html.

Bijou, S.W. (1982). Psicología del desarrollo infantil. La etapa básica de la niñez temprana. México: Trillas (Orig. 1976).

Bishop, D.V.M. (1989). Autism, Syndrome of Asperger and Semanticpragmatic Dysfunction: Where are the limits? Bristish Joumal o Disorders of Communication 24, 107-121.

Greer, R.D. (2002). Designing Teaching Strategies. An Applied Behavior. Analysis Systems Approach. Academic Press: San Diego, EE.UU.

Klin, A. y Volkmar, F. (1995). Asperger's Syndrome: Guidelimes for Assesment and Diagnosis. Disponible en Internet: http // www.apennj.org/guide.html.

Luciano, M.C. (1989). Una Aproximación Conceptual y Metodológica a las Alteraciones Conductuales en la Infancia. Universidad de Granada.

Luciano, M.C.; Herruzo, J y Barnes-Holmes, D. (2001). Generelization of say-do correpondence. The Psychological Record. 51, 111-130. Michael, J. (1982) Skinner's Elementory Verbal Relations: Some New Categones. The Analysis of Verbal Behavior. 1, 1-3.

Pérez-González, L.A. y Williams, G. (2002). Multicomponent Procedure to Teach Condicional Discriminations to Children with Autism. American Joumal on Mental Retardation. 107(4), 293-301.

Martos, J. (2001). Espectro autista: Una reflexión desde la práctica clírica. En J. Martos y A. Rivière (Comps.) Autismo: comprensión y 
explicación actual (pp. 121-153) Madrid. Ministerio de Trabajo y Asuntos Sociales.

Niwson, R. (2001). Vivir con autismo. En J. Martos yA. Rivière (Comps.). Autismo: comprensión y explicación actual (pp. 267-273). Madrid: Ministerio de Trabajo y Asuntos Sociales.

Rivière, A. (1997). Tratamiento y definición del espectro autista l.

Relaciones Sociales y Comunicación. En A. Rivière y J. Martos (Eds.). El tratamiento del autismo. Nuevas perspectivas. Madrid: INSERSO-APNA.

Skinner, B.F. (1981). Conducta verbal. México: Trillas (Orig. 1957). Williams, $G$ (1998). La rehabilitación de una niña con autismo y retraso mental severo. Comunicación presentada en el Cuarto Congreso Intemacional sobre Conductismo y Ciencias de la Conducta. Sevilla. Williams, G; Donley, C.R. y Keller, J.W. (2000). Teaching children with autism to ask questions about hidden objects. Joumal of Applied Behavior Analysis, 4 (33), 627-630.

Williams, K. (1995). Understanding the Student with Asperger's Syndrome: Guidelines for Teachers. Focus on Autistic Behavior, 10 (2). Disponible en Internet: www.aspennj.org/articles.html.

Wing, L. y Gould, J. (1979). Severe impairments of social interation and associated adnormalities in children: Epidemiology and classification. Joumal of Autism and Developmental Disorders, 9, 11-29. 